TRANSACTIONS OF THE

AMERICAN MATHEMATICAL SOCIETY

Volume 350, Number 4, April 1998, Pages 1467-1479

S 0002-9947(98)02099-6

\title{
BEREZIN'S QUANTIZATION ON FLAG MANIFOLDS AND SPHERICAL MODULES
}

\author{
ALEXANDER V. KARABEGOV
}

\begin{abstract}
We show that the theory of spherical Harish-Chandra modules naturally gives rise to Berezin's symbol quantization on generalized flag manifolds. It provides constructions of symbol algebras and of their representations for covariant and contravariant symbols, and also for symbols which so far have no explicit definition. For all these symbol algebras we give a general proof of the correspondence principle.
\end{abstract}

\section{INTRODUCTION}

Berezin's quantization on a symplectic manifold $\Omega$ (see [1]) is given by a family of associative algebras $\left\{\mathcal{A}_{h}\right\}$, whose elements are smooth functions on $\Omega$, together with their representations in Hilbert spaces $H_{h}$, where the parameter $h$ plays the role of the Planck constant. The multiplication $*_{h}$ in $\mathcal{A}_{h}$ must satisfy the so-called correspondence principle, as $h \rightarrow 0$. (A formal definition is given in Section 1).

Representation of the algebra $\mathcal{A}_{h}$ in $H_{h}$ maps a function $f \in \mathcal{A}_{h}$ to the operator $\hat{f}$ in $H_{h}$. In that case the function $f$ is called a symbol of the operator $\hat{f}$. The concrete examples of quantizations are usually based on explicit constructions of symbols (see [1], [2]).

In this paper we show how the theory of spherical Harish-Chandra modules naturally provides the algebras of covariant and contravariant symbols on generalized flag manifolds. Moreover, it also gives rise to the algebras of symbols which so far have no general construction or definition, as in the case of covariant and contravariant symbols. For all these symbol algebras we give a general proof of the correspondence principle. We also prove the conjecture from [3] on rational dependence of the product $f *_{h} g$ on $h$ on an arbitrary generalized flag manifold.

\section{The DEFinition of QUANTization}

We will give now a formal definition of quantization on a symplectic manifold $\Omega$. Let $F$ be a set of positive numbers with a limit point 0 . For each $h \in F$ let $\mathcal{A}_{h}$ be an algebra, which is a linear subspace of $C^{\infty}(\Omega)$, with a multiplication $*_{h}$ and a given representation in a Hilbert space $H_{h}$. Define $\mathcal{A}=\bigcup_{h} \mathcal{A}_{h}$ and let $\mathcal{A}$ be dense in $C^{\infty}(\Omega)$. These data determine a quantization on $\Omega$, if for $f, g \in \mathcal{A}$ and $h \rightarrow 0$ the correspondence principle holds,

$$
f *_{h} g \rightarrow f g, \quad h^{-1}\left(f *_{h} g-g *_{h} f\right) \rightarrow i\{f, g\}_{\Omega} .
$$

Received by the editors December 14, 1994 and, in revised form, June 14, 1996.

1991 Mathematics Subject Classification. Primary 58F06; Secondary 22E47.

Key words and phrases. Quantization, symbols, Harish-Chandra modules.

(C)1998 American Mathematical Society 
Here $\{\cdot, \cdot\}_{\Omega}$ is the Poisson bracket on $\Omega$. The limits in (1) make sense if for each $f \in \mathcal{A}$ one has $f \in \mathcal{A}_{h}$ for sufficiently small values of $h$ (a weak nesting property). This condition is valid if $\mathcal{A}_{h} \supset \mathcal{A}_{h^{\prime}}$ holds for $h<h^{\prime}$ (a strong nesting property).

\section{Covariant And CONTRAVARIANT Symbols}

Suppose a compact Lie group $K$ has an irreducible unitary representation in a $q$-dimensional vector space $E$ with a hermitian scalar product $\langle\cdot, \cdot\rangle, v \in E$ is a unit vector and $d k$ is the Haar measure on $K$ such that the total measure of $K$ is 1 . For $k \in K$ denote $v_{k}=k v$. Then the orthogonality relations for matrix coefficients immediately imply that for all $\xi, \eta \in E$ the Parseval identity holds:

$$
\langle\xi, \eta\rangle=q \int\left\langle\xi, v_{k}\right\rangle\left\langle v_{k}, \eta\right\rangle d k
$$

Thus the $K$-orbit of $v,\left\{v_{k}\right\}$, forms a supercomplete system of vectors in the sense of [2], and one can associate with it the constructions of covariant and contravariant symbols on $K$. Let $P_{k}$ be the orthogonal projection operator in $E$ to the vector $v_{k}$.

Definition. A function $f(k)$ on $K$ is called the covariant symbol of an operator $A \in$ End $E$, associated with the supercomplete system $\left\{v_{k}\right\}$, if $f(k)=\operatorname{tr} A P_{k}=$ $\left\langle A v_{k}, v_{k}\right\rangle$.

Such symbols for a compact semisimple Lie group $K$ were studied in [4].

Definition. A measurable function $g(k)$ on $K$ is called a contravariant symbol of an operator $B \in E n d E$, associated with the supercomplete system $\left\{v_{k}\right\}$, if $B=q \int g(k) P_{k} d k$.

The proofs of the following two lemmas are trivial.

Lemma 1. A measurable function $g(k)$ is a contravariant symbol of an operator $B \in$ End $E$ iff for any operator $A \in$ End $E$ with the covariant symbol $f(k)$ the equality $q \int f(k) g(k) d k=\operatorname{tr} A B$ holds.

Let $k_{0} \in K$. Denote $v^{\prime}=k_{0} v$ and consider the supercomplete system $\left\{v_{k}^{\prime}=k v^{\prime}\right\}$.

Lemma 2. Let $f(k)$ be the covariant (contravariant) symbol of an operator $A \in$ End $E$, associated with the supercomplete system $\left\{v_{k}\right\}$. Then $f\left(k k_{0}\right)$ is the covariant (contravariant) symbol of $A$, associated with the supercomplete system $\left\{v_{k}^{\prime}\right\}$.

\section{A SPHERICAL PRINCIPAL SERIES OF HARISH-CHANDRA MODUleS}

We give a construction of a spherical principal series of Harish-Chandra modules, following [5].

Let $g_{c}$ be a complex semisimple Lie algebra, $h_{c}$ its Cartan subalgebra, $W$ the Weyl group, $\triangle, \triangle^{+}, \Sigma$ the sets of all nonzero, positive and simple roots respectively, $\rho$ the half-sum of positive roots. For each $\alpha \in \triangle$ choose weight elements $X_{\alpha} \in g_{c}$ such that $\left[H_{\alpha}, X_{ \pm \alpha}\right]= \pm 2 X_{ \pm \alpha}$ for $H_{\alpha}=\left[X_{\alpha}, X_{-\alpha}\right]$. An element $\lambda \in h_{c}^{*}$ is called dominant if $\lambda\left(H_{\alpha}\right) \geq 0$ for all $\alpha \in \Sigma$, and is a weight if $\lambda\left(H_{\alpha}\right) \in \mathbf{Z}$ for all $\alpha \in \Sigma$. Denote $n_{c}, n_{c}^{-}$the complex subalgebras of $g_{c}$ spanned by $\left\{X_{\alpha}\right\}$ for $\alpha$ running over $\Delta^{+},-\Delta^{+}$respectively. Then $g_{c}=n_{c}^{-} \oplus h_{c} \oplus n_{c}$ is the Gauss decomposition.

Let $g_{r}, h_{r}, n_{r}, n_{r}^{-}$denote the Lie algebras $g_{c}, h_{c}, n_{c}$ and $n_{c}^{-}$respectively, considered as real Lie algebras, and let $\theta$ be an automorphism of $g_{r}$, antilinear with respect to the complex structure on $g_{c}$ and such that $\theta X_{\alpha}=-X_{-\alpha}, \theta H_{\alpha}=-H_{\alpha}$. 
The subspace of $\theta$-invariant elements of $g_{r}, k_{r}=g_{r}^{\theta}$, is a compact form of $g_{c}$. Denote by $a_{r}$ the subalgebra of $g_{r}$ generated by $\left\{H_{\alpha}\right\}$. Then $g_{r}=k_{r} \oplus a_{r} \oplus n_{r}$ is the Iwasawa decomposition.

Now let $G$ be the complex semisimple connected simply connected Lie group with the Lie algebra $g_{r}$, considered as a real Lie group, and $G=K A N$ be the corresponding Iwasawa decomposition. Then $K$ is a compact semisimple connected simply connected Lie group. Let $m_{r}=k_{r} \cap h_{r}, M$ the maximal torus in $K$ with the Lie algebra $m_{r}, M^{\prime}$ its normalizer in $K$, so that $W=M^{\prime} / M$.

Denote by $\Lambda$ the space of functions on $K, K$-finite with respect to the left shifts and invariant with respect to the right shifts by the elements of $M$ (in [5] it is denoted by $L(0))$. Let $X \in a_{r}, a=\exp X \in A$. Then for $\lambda \in h_{c}^{*}$ we have $a^{\lambda}=\mathrm{e}^{\langle\lambda, X\rangle}$. Denote by $\tilde{\Lambda}(\lambda)$ the space of functions $f$ on $G$ such that for $g \in G, m \in$ $M, a \in A, n \in N$ we have $f($ gman $)=f(g) a^{2(\lambda-\rho)}$, and the restriction of $f$ to $K$ belongs to $\Lambda$ (in the notation of [5], $L(0,-2 \lambda)$ ). Define a $\left(g_{r}, K\right)$-module structure on $\tilde{\Lambda}(\lambda)$, for $f \in \tilde{\Lambda}(\lambda), X \in g_{r}, k \in K$, by $X f(g)=\left.\frac{d}{d t}\right|_{t=0} f(\exp (-t X) g), k f(g)=$ $f\left(k^{-1} g\right)$. The module $\tilde{\Lambda}(\lambda)$ is spherical, i.e., its subspace of $K$-invariant vectors is one dimensional.

By virtue of the Iwasawa decomposition, the restriction to $K$ determines a bijection of $\tilde{\Lambda}(\lambda)$ onto $\Lambda$. Denote by $\Lambda(\lambda)$ the space $\Lambda$, endowed with a $\left(g_{r}, K\right)$-module structure, induced from $\tilde{\Lambda}(\lambda)$. The $\left(g_{r}, K\right)$-modules $\Lambda(\lambda)$ (or $\left.\tilde{\Lambda}(\lambda)\right)$ form the spherical principal series of Harish-Chandra $\left(g_{r}, K\right)$-modules.

Let $A(\lambda)$ and $B(\lambda)$ denote respectively the cyclic submodule of $\Lambda(\lambda)$ generated by $1 \in \Lambda(\lambda)$, and its maximal proper submodule. Then $\hat{\Lambda}(\lambda)=A(\lambda) / B(\lambda)$ is the canonical simple subquotient of the principal series. It is known, that for a given $\lambda \in h_{c}^{*}$ the modules $\hat{\Lambda}(w \lambda), w \in W$, are isomorphic to each other (see [5]).

\section{An algebra structure on $A(\lambda+\rho)$}

Let $\mathcal{U}\left(g_{c}\right)$ be the universal enveloping algebra of $g_{c}$. Define an action of $g_{r}$ on $\mathcal{U}\left(g_{c}\right)$ : for $X \in g_{r}, u \in \mathcal{U}\left(g_{c}\right)$ let $X: u \mapsto \theta(X) u-u X$, and let $K$ act on $\mathcal{U}\left(g_{c}\right)$ by the adjoint action (denoted by $A d$ ). If $X \in k_{r}$, then $\theta X=X$, so $X: u \mapsto X u-u X$, i.e., $k_{r}$ acts on $\mathcal{U}\left(g_{c}\right)$ by the adjoint action. Thus the actions of $g_{r}$ and $K$ agree on $k_{r}$ and $\mathcal{U}\left(g_{c}\right)$ is a $\left(g_{r}, K\right)$-module.

Consider a morphism of $\left(g_{r}, K\right)$-modules $s_{\lambda}: \mathcal{U}\left(g_{c}\right) \rightarrow \Lambda(\lambda+\rho)$ such that $s_{\lambda} 1=1$. This requirement defines $s_{\lambda}$ uniquely, since $1 \in \mathcal{U}\left(g_{c}\right)$ is cyclic. Thus $s_{\lambda}$ coincides with the morphism $\Phi_{-\lambda}$, introduced in [6], Sect. 9.6.5. Let $L(\lambda)$ denote the simple highest weight $g_{c}$-module of weight $\lambda-\rho$, and Ann $L(\lambda)$ its annihilator.

Lemma 3. (i) $\operatorname{Im} s_{\lambda}=A(\lambda+\rho)$; (ii) $\operatorname{Ker} s_{\lambda}=\operatorname{Ann} L(\lambda+\rho)$.

The proof of (i) is trivial, and (ii) is proved in lemma 9.6.5 of [6].

Corollary. $A(\lambda+\rho)$ carries the structure of an algebra naturally isomorphic to $\mathcal{U}\left(g_{c}\right) /$ Ann $L(\lambda+\rho)$.

We will give now an explicit construction of the mapping $s_{\lambda}$. For $\lambda \in h_{c}^{*}$ define a functional $\varphi_{\lambda}$ on $\mathcal{U}\left(g_{c}\right)$ (in the notation of [6], Sect.9.6.4., it corresponds to $\varphi_{-\lambda}$ ). It follows from the Gauss decomposition, that

$$
\mathcal{U}\left(g_{c}\right)=\left(n_{c}^{-} \mathcal{U}\left(g_{c}\right)+\mathcal{U}\left(g_{c}\right) n_{c}\right) \oplus \mathcal{U}\left(h_{c}\right) .
$$


Denote the projection of $u \in \mathcal{U}\left(g_{c}\right)$ on $\mathcal{U}\left(h_{c}\right)$ by $u_{0}$. Since $\mathcal{U}\left(h_{c}\right)$ is naturally isomorphic to the symmetric algebra $S\left(h_{c}\right)$ (or to the algebra of polynomials on $h_{c}^{*}$ ), one can evaluate $u_{0}$ at the point $\lambda \in h_{c}^{*}$. Define $\varphi_{\lambda}(u)=u_{0}(\lambda)$.

For $u \in \mathcal{U}\left(g_{c}\right)$ define a function $\tilde{s}_{\lambda} u$ on $G$ by the formula

$$
\tilde{s}_{\lambda} u(k a n)=\varphi_{\lambda}\left(A d\left(k^{-1}\right) u\right) a^{2 \lambda}
$$

where $k \in K, a \in A, n \in N$.

Proposition 1. The mapping $\tilde{s}_{\lambda}: u \mapsto \tilde{s}_{\lambda} u$ is a morphism of $\left(g_{r}, K\right)$-modules from $\mathcal{U}\left(g_{c}\right)$ to $\tilde{\Lambda}(\lambda+\rho)$.

Proof. The $K$-equivariance of $\tilde{s}_{\lambda}$ can be checked immediately. Now the $K$-finiteness of $\tilde{s}_{\lambda} u$ follows from the finiteness of the adjoint action of $K$ in $\mathcal{U}\left(g_{c}\right)$. The adjoint action of $M$ on $\mathcal{U}\left(g_{c}\right)$ preserves the decomposition (2) and is trivial on $\mathcal{U}\left(h_{c}\right)$, which implies the invariance of $\tilde{s}_{\lambda} u$ with respect to the right shifts by the elements of $M$. Thus $\tilde{s}_{\lambda} u \in \tilde{\Lambda}(\lambda+\rho)$. It remains to show that $\tilde{s}_{\lambda}$ is $g_{r}$-equivariant, that is, for $X \in g_{r}, u \in \mathcal{U}\left(g_{c}\right)$ the functions $X \tilde{s}_{\lambda} u$ and $\tilde{s}_{\lambda}(\theta(X) u-u X)$ coincide. Since the restriction of $\tilde{\Lambda}(\lambda+\rho)$ to $K$ is a bijection onto $\Lambda(\lambda+\rho)$ and $\tilde{s}_{\lambda}$ is $K$-equivariant, it is enough to compare these functions at $e \in K$, that is, to check the equality

$$
\left.\frac{d}{d t}\right|_{t=0} \tilde{s}_{\lambda} u(\exp (-t X))=\varphi_{\lambda}(\theta(X) u-u X)
$$

for $X \in k_{r}, a_{r}, n_{r}$. Since the actions of $g_{r}$ and $K$ agree on $k_{r}$, (3) holds for $X \in k_{r}$. Let $X \in a_{r}$; then $\tilde{s}_{\lambda} u(\exp (-t X))=\mathrm{e}^{-2 t\langle\lambda, X\rangle} \varphi_{\lambda}(u)$, so the left-hand side of (3) equals $-2\langle\lambda, X\rangle \varphi_{\lambda}(u)$. On the other hand, $\theta X=-X$ for $X \in a_{r}$, so $\theta(X) u-u X=$ $-2 u X$. Since the multiplication by $X \in a_{r}$ preserves the decomposition (2), one has $\varphi_{\lambda}(-2 u X)=-2\langle\lambda, X\rangle \varphi_{\lambda}(u)$. Now let $X \in n_{r}$. Then $\tilde{s}_{\lambda} u(\exp (-t X))=\varphi_{\lambda}(u)$ does not depend on $t$, and thus the left-hand side of (3) is equal to 0 . Since $\theta X \in n_{r}^{-}$, therefore $\theta(X) u-u X$ belongs to the sum in the parenthesis in (2), so the right-hand side of (3) is also 0 . This concludes the proof.

Corollary. The mapping $s_{\lambda}$ is given by the formula $s_{\lambda} u(k)=\varphi_{\lambda}\left(\operatorname{Ad}\left(k^{-1}\right) u\right)$.

Proof. Since the restriction of $\tilde{s}_{\lambda} 1$ to $K$ equals the constant 1 , the morphism $\mathcal{U}\left(g_{c}\right) \ni$ $\left.u \mapsto \tilde{s}_{\lambda} u\right|_{K} \in \Lambda(\lambda+\rho)$ coincides with $s_{\lambda}$. Now the proof follows from the definition of $\tilde{s}_{\lambda}$.

\section{Symbol ALGebras}

Let $\lambda \in h_{c}^{*}$ be a dominant weight. Consider the holomorphic irreducible representation of the group $G$ with the highest weight $\lambda$ in the space $E^{\lambda}$ with a $K$-invariant hermitian scalar product $\langle\cdot, \cdot\rangle$, and a highest weight vector $v$ of length 1 . Denote the corresponding representation of $\mathcal{U}\left(g_{c}\right)$ in $E^{\lambda}$ by $\pi_{\lambda}$. Let $w_{0} \in W$ be the Coxeter element (the element of the maximal reduced length, which maps $\Delta^{+}$to $-\Delta^{+}$). Set $\lambda^{\prime}=-w_{0} \lambda$. It is known, that the module $E^{\lambda^{\prime}}$ is dual to $E^{\lambda}$.

Endow the space End $E^{\lambda}$ with a $\left(g_{r}, K\right)$-module structure. For $A \in E n d E^{\lambda}$, $X \in g_{r}$ and $k \in K$, let $X: A \mapsto \pi_{\lambda}(\theta X) A-A \pi_{\lambda}(X)$ and $k: A \mapsto k A k^{-1}$.

The complexification of the algebra $g_{r}$ is isomorphic to $g_{c} \times g_{c}$. Consider an imbedding of $g_{r}$ in $g_{c} \times g_{c}, X \mapsto(\theta X, X)$. Then End $E^{\lambda}$ is isomorphic to $E^{\lambda} \otimes E^{\lambda^{\prime}}$ as a $g_{c} \times g_{c}$-module, and therefore is simple.

It is easy to check that $\pi_{\lambda}: \mathcal{U}\left(g_{c}\right) \rightarrow E n d E^{\lambda}$ is a surjective morphism of $\left(g_{r}, K\right)$-modules. 
Proposition 2. Let $\lambda \in h_{c}^{*}$ be a dominant weight, $u \in \mathcal{U}\left(g_{c}\right)$. The function $s_{\lambda} u$ is the covariant symbol of the operator $\pi_{\lambda}(u) \in E$ End $E^{\lambda}$, associated with the supercomplete system $\left\{v_{k}=k v\right\}$.

Proof. Suppose $A d\left(k^{-1}\right) u$ is decomposed according to (2), $A d\left(k^{-1}\right) u=u_{0}+X u_{1}+$ $u_{2} Y$, where $u_{0} \in \mathcal{U}\left(h_{c}\right), X \in n_{c}^{-}, Y \in n_{c}$. Let us calculate the covariant symbol of the operator $\pi_{\lambda}(u)$, associated with $\left\{v_{k}\right\}$ :

$$
\begin{aligned}
\left\langle\pi_{\lambda}(u) v_{k}, v_{k}\right\rangle & =\left\langle\pi_{\lambda}\left(A d\left(k^{-1}\right) u\right) v, v\right\rangle \\
& =\left\langle\pi_{\lambda}\left(u_{0}\right) v, v\right\rangle+\left\langle\pi_{\lambda}\left(X u_{1}\right) v, v\right\rangle+\left\langle\pi_{\lambda}\left(u_{2} Y\right) v, v\right\rangle .
\end{aligned}
$$

Since $v$ is the highest weight vector of the weight $\lambda$, the first summand in the righthand side of (4) is equal to $s_{\lambda}(k)$, and the third one is 0 . Finally, since $\theta X \in n_{c}$, we have $\left\langle\pi_{\lambda}\left(X u_{1}\right) v, v\right\rangle=-\left\langle\pi_{\lambda}\left(u_{1}\right) v, \pi_{\lambda}(\theta X) v\right\rangle=0$.

The proposition is proved.

Denote by $\zeta$ the mapping which maps an operator $A \in E n d E^{\lambda}$ to its covariant symbol, $\zeta A(k)=\langle A k v, k v\rangle$.

Corollary. $\zeta$ is an isomorphism of the $\left(g_{r}, K\right)$-modules and associative algebras End $E^{\lambda}$ and $A(\lambda+\rho)$.

Proof. Proposition 2 asserts that $s_{\lambda}=\zeta \pi_{\lambda}$. It follows from the surjectivity of $\pi_{\lambda}$ and from the fact that the image of $s_{\lambda}$ coincides with $A(\lambda+\rho)$, that the image of $\zeta$ coincides with $A(\lambda+\rho)$ as well. The mapping $\zeta$ is injective since End $E^{\lambda}$ is a simple module. Finally, since $s_{\lambda}$ and $\pi_{\lambda}$ are morphisms of $\left(g_{r}, K\right)$-modules and homomorphisms of associative algebras, the same is true for $\zeta$.

The shifted action of the Weyl group $W$ on $h_{c}^{*}$ is defined as follows. For $\lambda \epsilon$ $h_{c}^{*}, w \in W$ we put $w \cdot \lambda=w(\lambda+\rho)-\rho$. For a dominant weight $\lambda \in h_{c}^{*}$, the $\left(g_{r}, K\right)$ module $\hat{\Lambda}(\lambda+\rho)=A(\lambda+\rho) / B(\lambda+\rho)$, together with all $\hat{\Lambda}(w(\lambda+\rho)), w \in W$, are isomorphic to End $E^{\lambda}$. This implies that, since the modules $A(w(\lambda+\rho))$, $w \in W$, and End $E^{\lambda}$ are spherical, one can choose a surjective morphism $\tau_{w \cdot \lambda}$ : $A(w(\lambda+\rho)) \rightarrow$ End $E^{\lambda}$ such that $\tau_{w \cdot \lambda} 1=1$. It is clear, that $\tau_{w \cdot \lambda} s_{w \cdot \lambda}=\pi_{\lambda}$, and the $\left(g_{r}, K\right)$-module morphism $\tau_{w \cdot \lambda}$ is also an algebra homomorphism, i.e. a representation of $A(w(\lambda+\rho))$ in $E^{\lambda}$.

Now we can introduce the following

Definition. A function $f \in A(w(\lambda+\rho))$ is called a mixed symbol of an operator $A \in$ End $E^{\lambda}$, corresponding to the element $w \in W$, if $A=\tau_{w \cdot \lambda} f$.

Let $\mu \in h_{c}^{*}, f \in \Lambda(\mu), g \in \Lambda(-\mu)$, and $q$ be a nonzero constant. It is known that $(f, g)_{q}=q \int_{K} f(k) g(k) d k$ is a pairing of $\left(g_{r}, K\right)$-modules $\Lambda(\mu)$ and $\Lambda(-\mu)$ (see [5]). Since the module $B(\mu)$ does not contain $1 \in A(\mu)$, it is orthogonal to $1 \in A(-\mu)$, and therefore to the whole module $A(-\mu)$, with respect to $(\cdot, \cdot)_{q}$. Besides, $(1,1)_{q}=q$. This implies that $(\cdot, \cdot)_{q}$ induces a nondegenerate pairing of the simple $\left(g_{r}, K\right)$-modules $\hat{\Lambda}(\mu)$ and $\hat{\Lambda}(-\mu)$, and each pairing of these modules can be obtained by choosing the appropriate value of $q$.

Now let $\lambda \in h_{c}^{*}$ be a dominant weight. The module $\hat{\Lambda}(\lambda+\rho)$ is isomorphic to End $E^{\lambda}$ and dual to $\hat{\Lambda}(-\lambda-\rho)$. Since $-\lambda-\rho=w_{0}\left(\lambda^{\prime}+\rho\right)$, the module $\hat{\Lambda}(-\lambda-\rho)=\hat{\Lambda}\left(w_{0}\left(\lambda^{\prime}+\rho\right)\right)$ is isomorphic to End $E^{\lambda^{\prime}}$. On the other hand, since the $g_{c}$-modules $E^{\lambda}$ and $E^{\lambda^{\prime}}$ are dual, an operator $A \in E n d E^{\lambda}$ has the transpose $A^{t} \in E n d E^{\lambda^{\prime}}$. It is easy to check, that for $A \in E n d E^{\lambda}$ and $B \in E n d E^{\lambda^{\prime}}, \operatorname{tr} A B^{t}$ 
also defines a nondegenerate pairing of the $\left(g_{r}, K\right)$-modules $E n d E^{\lambda}$ and $E n d E^{\lambda^{\prime}}$. This implies

Lemma 4. Let $f \in A(\lambda+\rho), g \in A\left(w_{0}\left(\lambda^{\prime}+\rho\right)\right)$, and $q$ be the dimension of $E^{\lambda}$. Then

$$
q \int_{K} f(k) g(k) d k=\operatorname{tr}\left(\tau_{\lambda} f\right)\left(\tau_{w_{0} \cdot \lambda^{\prime}} g\right)^{t} .
$$

To prove the assertion of the lemma it is enough to check it for $f=g=1$.

It follows from Proposition 2, that a function $f$ is the covariant symbol of the operator $\tau_{\lambda} f$, associated with the supercomplete system $\left\{v_{k}=k v\right\}$. Now Lemma 1 and Lemma 4 imply that a function $g$ is a contravariant symbol of the operator $\left(\tau_{w_{0} \cdot \lambda^{\prime}} g\right)^{t}$, also associated with the supercomplete system $\left\{v_{k}\right\}$. Taking into account that for a dominant weight $\lambda$ we have $\tau_{w \cdot \lambda} s_{w \cdot \lambda}=\pi_{\lambda}$, we get the following:

Lemma 5. For $u \in \mathcal{U}\left(g_{c}\right)$ the function $s_{w_{0} \cdot \lambda^{\prime}} u$ is a contravariant symbol of the operator $\left(\pi_{\lambda^{\prime}}(u)\right)^{t} \in$ End $E^{\lambda}$, associated with the supercomplete system $\left\{v_{k}\right\}$.

Let $u \mapsto \check{u}$ be an involutive anti-automorphism of $\mathcal{U}\left(g_{c}\right)$, such that for $X \in g_{c}$ we have $\check{X}=-X$. Since the $g_{c}$-modules $E^{\lambda}$ and $E^{\lambda^{\prime}}$ are dual, the transpose operator to $\pi_{\lambda^{\prime}}(X) \in$ End $E^{\lambda^{\prime}}$ is equal to $-\pi_{\lambda}(X) \in E n d E^{\lambda}$, which implies that $\left(\pi_{\lambda^{\prime}}(u)\right)^{t}=\pi_{\lambda}(\check{u})$. Therefore it follows from Lemma 5 , that the function $s_{w_{0} \cdot \lambda^{\prime}} u$ is a contravariant symbol of the operator $\pi_{\lambda}(\check{u})$, associated with $\left\{v_{k}\right\}$.

Lemma 6. Let $\tilde{w}_{0} \in M^{\prime}$ be a representative of the Coxeter element $w_{0} \in W$, $u \in \mathcal{U}\left(g_{c}\right)$. Then $s_{w_{0} \cdot \lambda^{\prime}} u(k)=s_{w_{0} \cdot \lambda} \check{u}\left(k \tilde{w}_{0}^{-1}\right)$.

Proof. Since the mappings $u \mapsto \check{u}$ and $u \mapsto s_{\lambda} u$ are $K$-equivariant, it is enough to check the equality of the lemma at the point $k=e$. Let $u$ be decomposed in accordance with (2), $u=u_{0}+X u_{1}+u_{2} Y$, where $u_{0} \in \mathcal{U}\left(h_{c}\right), X \in n_{c}^{-}, Y \in n_{c}$. Then, taking into account that $w_{0} \cdot \lambda^{\prime}=-\lambda-2 \rho$, we get $s_{w_{0} \cdot \lambda^{\prime}} u(e)=u_{0}(-\lambda-2 \rho)$. Since the anti-automorphism $u \mapsto \check{u}$ for $u \in \mathcal{U}\left(h_{c}\right)$ reduces to the change of a sign in the argument when $\mathcal{U}\left(h_{c}\right)$ is identified with $S\left(h_{c}\right)$, then $u_{0}(-\lambda-2 \rho)=\check{u}_{0}(\lambda+2 \rho)$. On the other hand, $\breve{u}=\check{u}_{0}-\breve{u}_{1} X-Y \check{u}_{2}$, so

$$
\operatorname{Ad}\left(\tilde{w}_{0}\right) \check{u}=\operatorname{Ad}\left(\tilde{w}_{0}\right) \check{u}_{0}-\operatorname{Ad}\left(\tilde{w}_{0}\right)\left(\check{u}_{1} X+Y \check{u}_{2}\right) .
$$

Since $\operatorname{Ad}\left(\tilde{w}_{0}\right) X \in n_{c}$, and $\operatorname{Ad}\left(\tilde{w}_{0}\right) Y \in n_{c}^{-}$, the subtrahend in the right-hand side of (5) belongs to the sum in the parenthesis in (2), therefore

$$
s_{w_{0} \cdot \lambda} \check{u}\left(\tilde{w}_{0}^{-1}\right)=\varphi_{w_{0} \cdot \lambda}\left(A d\left(\tilde{w}_{0}\right) \check{u}\right)=\left(A d\left(\tilde{w}_{0}\right) \check{u}_{0}\right)\left(w_{0} \cdot \lambda\right)=\check{u}_{0}\left(w_{0}\left(w_{0} \cdot \lambda\right)\right) .
$$

To conclude the proof it is enough to notice that $w_{0}\left(w_{0} \cdot \lambda\right)=\lambda+2 \rho$.

Consider a vector $v^{\prime}=\tilde{w}_{0} v \in E^{\lambda}$. Since $v \in E^{\lambda}$ is the highest weight vector, then $v^{\prime}$ is the lowest weight one.

Theorem 1. (i) A mixed symbol of an operator $A \in E n d E^{\lambda}$, corresponding to the unit element $e \in W$, is the covariant symbol of $A$, associated with the supercomplete system $\left\{v_{k}=k v\right\}$.

(ii) A mixed symbol of an operator $A$, corresponding to the element $w_{0} \in W$, is a contravariant symbol of $A$, associated with the supercomplete system $\left\{v_{k}^{\prime}=k v^{\prime}\right\}$.

Proof. The assertion (i) is proved in Proposition 2. It follows from Lemmas 5 and 6 that for $u \in \mathcal{U}\left(g_{c}\right)$ the function $s_{w_{0} \cdot \lambda} u\left(k \tilde{w}_{0}^{-1}\right)$ is a contravariant symbol of the operator $\pi_{\lambda}(u)$, associated with the supercomplete system $\left\{v_{k}\right\}$. Now it 
follows from Lemma 2, that the function $s_{w_{0} \cdot \lambda} u(k)$ is a contravariant symbol of the operator $\pi_{\lambda}(u)$, associated with the supercomplete system $\left\{v_{k}^{\prime}\right\}$, which concludes the proof.

\section{Symbol ALGEBRAS ON FlaG MANIFOLDS}

Denote by $a_{r}^{*}$ the real subspace of $h_{c}^{*}$, formed by those functionals which are real on $a_{r}$. In particular, the roots and weights of $g_{c}$ belong to $a_{r}^{*}$. We will give a condition on $\lambda \in a_{r}^{*}$, under which one can define a pushforward of the algebra $A(\lambda+\rho)$ to an orbit of the adjoint representation of $K$ in $k_{r}$. These orbits are generalized flag manifolds.

The Killing form $(\cdot, \cdot)$ on $g_{c}$ is positive definite on $a_{r}$ and negative definite on $m_{r}$. Let $\lambda \in a_{r}^{*}$. Define a mapping $\Psi_{\lambda}: K \mapsto g_{c}$ such that for each $k \in K$ and $X \in g_{c}$ we have $i s_{\lambda} X(k)=\left(X, \Psi_{\lambda}(k)\right)$. The following lemma is straightforward.

Lemma 7. For $k, l \in K, \Psi_{\lambda}\left(k^{-1} l\right)=A d(k) \Psi_{\lambda}(l)$.

The lemma asserts that $\Psi_{\lambda}$ is a $K$-equivariant mapping. For each $\lambda \in a_{r}^{*}$ choose $H^{\lambda} \in a_{r}$ such that $\lambda(H)=\left(H, H^{\lambda}\right)$ for $H \in h_{c}$. Notice, that $H^{\alpha}=$ $\left(2 /\left(H_{\alpha}, H_{\alpha}\right)\right) H_{\alpha}$ for $\alpha \in \triangle$. Since $i a_{r}=m_{r}$, for $\lambda \in a_{r}^{*}$ we have $i H^{\lambda} \in m_{r}$.

Lemma 8. Let $\lambda \in a_{r}^{*}$. Then $\Psi_{\lambda}(e)=i H^{\lambda}$, so the image of $\Psi_{\lambda}$ is the orbit of the point $i H^{\lambda} \in m_{r}$ with respect to the adjoint action of $K$ on $g_{c}$, and it lies completely in $k_{r}$.

Proof. For any $X \in g_{c}$ we have $\left(X, \Psi_{\lambda}(e)\right)=i s_{\lambda} X(e)=i \varphi_{\lambda}(X)$. If $X \in n_{c} \oplus n_{c}^{-}$, then $i \varphi_{\lambda}(X)=0$ and $\left(X, H^{\lambda}\right)=0$. And if $X \in h_{c}$, then $i \varphi_{\lambda}(X)=i \lambda(X)=$ $\left(X, i H^{\lambda}\right)$. Thus $\left(X, \Psi_{\lambda}(e)\right)=\left(X, i H^{\lambda}\right)$ holds for each $X \in g_{c}$, which implies the assertion of the lemma.

Denote by $\triangle(\lambda)$ the set of roots $\alpha \in \triangle$ such that $\lambda\left(H_{\alpha}\right)=0$ for $\lambda \in a_{r}^{*}$, and let $\triangle^{+}(\lambda)=\triangle^{+} \cap \triangle(\lambda)$. It is evident, that $\lambda\left(H_{\alpha}\right)=0$ iff $\lambda\left(H^{\alpha}\right)=0$.

The centralizer of $H^{\lambda}$ in $g_{c}$ is generated by the Cartan subalgebra $h_{c}$ and the elements $X_{\alpha}, \alpha \in \triangle(\lambda)$. Denote by $k_{r}^{\lambda} \subset k_{r}$ the centralizer of $i H^{\lambda}$ in $k_{r}$. Then $k_{r}^{\lambda}$ is generated by the subalgebra $m_{r}$ and the elements $X_{\alpha}-X_{-\alpha}$ and $i\left(X_{\alpha}+X_{-\alpha}\right), \alpha \in$ $\triangle^{+}(\lambda)$. Since the stabilizer $K^{\lambda} \subset K$ of the element $i H^{\lambda} \in k_{r}$ with respect to the adjoint action of $K$ is connected, it is the integral subgroup of the subalgebra $k_{r}^{\lambda} \subset k_{r}$. The orbit $\Omega_{\lambda} \subset k_{r}$ of the point $i H^{\lambda}$ under the adjoint action of $K$ is isomorphic to $K / K^{\lambda}$ as a homogeneous space, and is a generalized flag manifold.

Let $\sigma \subset \Sigma$ be a set of simple roots. Denote by $\langle\sigma\rangle$ the set of roots which are linear combinations of elements of $\sigma$.

Definition. An element $\lambda \in a_{r}^{*}$ is called regular relative to $\sigma \subset \Sigma$, if $\triangle(\lambda)=\langle\sigma\rangle$.

Definition. An element $\lambda \in a_{r}^{*}$ is called relatively regular, if it is regular relative to some $\sigma \subset \Sigma$.

Lemma 9. An element $\lambda \in a_{r}^{*}$ is relatively regular iff for all $\alpha, \beta \in \Delta^{+}$such that $\alpha+\beta \in \triangle^{+}(\lambda)$ we have $\alpha, \beta \in \triangle^{+}(\lambda)$.

Proof. The necessity is evident. Let us prove the sufficiency. Set $\sigma=\Sigma \cap \triangle(\lambda)$. We will show that $\triangle(\lambda)=\langle\sigma\rangle$. Since $\triangle(\lambda)=-\triangle(\lambda)$, it is enough to consider elements $\gamma \in \Delta^{+}(\lambda)$. If $\gamma \in \Delta^{+}$, then either $\gamma \in \Sigma$, or one can find $\alpha \in \Delta^{+}, \beta \in \Sigma$ such that $\gamma=\alpha+\beta$. It then follows from $\gamma \in \triangle^{+}(\lambda)$ that either $\gamma \in \sigma$, or $\alpha \in \triangle^{+}(\lambda)$, 
and $\beta \in \sigma$. It remains to use the induction over the number of summands in the decomposition of $\gamma$ into a sum of simple roots.

Lemma 10. Any dominant element $\lambda \in a_{r}^{*}$ is relatively regular.

Since the Killing form is positive definite on $a_{r}, \lambda \in a_{r}^{*}$ is dominant iff for all $\alpha \in \triangle^{+}$we have $\lambda\left(H^{\alpha}\right) \geq 0$. Let $\alpha, \beta \in \Delta^{+}$be such that $\gamma=\alpha+\beta \in \Delta^{+}(\lambda)$. Then $\lambda\left(H^{\alpha}\right)+\lambda\left(H^{\beta}\right)=\lambda\left(H^{\gamma}\right)=0$, and since $\lambda\left(H^{\alpha}\right) \geq 0, \lambda\left(H^{\beta}\right) \geq 0$, then $\lambda\left(H^{\alpha}\right)=\lambda\left(H^{\beta}\right)=0$, which means that $\alpha, \beta \in \Delta^{+}(\lambda)$. The lemma follows now from Lemma 9.

Lemma 11. All the elements of $A(\lambda+\rho), \lambda \in a_{r}^{*}$, are invariant under the right shifts by the elements of $K^{\lambda}$ only if $\lambda$ is relatively regular.

Proof. Assume $\alpha, \beta \in \triangle^{+}$are such that $\gamma=\alpha+\beta \in \triangle^{+}(\lambda)$, and, say, $\alpha \notin \triangle^{+}(\lambda)$. We will show that the function $s_{\lambda} X_{\alpha} X_{\beta}$ is not constant on $K^{\lambda} \subset K$. In order to do that, let us calculate the infinitesimal action of the element $X_{\gamma}-X_{-\gamma} \in k_{r}^{\lambda}$ on $s_{\lambda} X_{\alpha} X_{\beta}$ at the point $e \in K$ :

$$
\begin{aligned}
& \left.\frac{d}{d t}\right|_{t=0}\left(s_{\lambda} X_{\alpha} X_{\beta}\right)\left(\exp \left(-t\left(X_{\gamma}-X_{-\gamma}\right)\right)\right) \\
& \quad=\left.\frac{d}{d t}\right|_{t=0} \varphi_{\lambda}\left(A d\left(\exp t\left(X_{\gamma}-X_{-\gamma}\right)\right) X_{\alpha} X_{\beta}\right)=\varphi_{\lambda}\left(\left[X_{\gamma}-X_{-\gamma}, X_{\alpha} X_{\beta}\right]\right) .
\end{aligned}
$$

Since $X_{-\gamma} X_{\alpha} X_{\beta}, X_{\gamma} X_{\alpha} X_{\beta}$ and $X_{\alpha} X_{\beta} X_{\gamma}$ belong to the kernel of $\varphi_{\lambda}$, the expression in (6) is equal to $\varphi_{\lambda}\left(X_{\alpha} X_{\beta} X_{-\gamma}\right)=\varphi_{\lambda}\left(X_{\alpha} X_{-\gamma} X_{\beta}\right)+\varphi_{\lambda}\left(X_{\alpha}\left[X_{\beta}, X_{-\gamma}\right]\right)$. Since $\beta-\gamma=-\alpha \in \triangle$, it follows that $\left[X_{\beta}, X_{-\gamma}\right]=c \cdot X_{-\alpha}$, where $c$ is a nonzero constant. Finally, the expression in (6) is equal to $\varphi_{\lambda}\left(X_{\alpha}\left[X_{\beta}, X_{-\gamma}\right]\right)=c \varphi_{\lambda}\left(X_{\alpha} X_{-\alpha}\right)=$ $c \varphi_{\lambda}\left(\left[X_{\alpha}, X_{-\alpha}\right]\right)=c \varphi_{\lambda}\left(H_{\alpha}\right)=c \lambda\left(H_{\alpha}\right) \neq 0$, which implies the assertion of the lemma.

Let us now show that the inverse to Lemma 11 is also true.

Let $\lambda \in a_{r}^{*}$ be a dominant weight, $\pi_{\lambda}$ the representation of $\mathcal{U}\left(g_{c}\right)$ in the module $E^{\lambda}$ with the highest weight $\lambda$ and the highest weight vector $v$ of length 1 .

Lemma 12. Let $\alpha \in \triangle^{+}(\lambda)$. Then $\pi_{\lambda}\left(X_{-\alpha}\right) v=0$.

Proof. Consider $E^{\lambda}$ as a module over the $s l_{2}$-subalgebra of $g_{c}$ generated by the elements $X_{-\alpha}, H_{\alpha}, X_{\alpha}$. Assume $\pi_{\lambda}\left(X_{-\alpha}\right) v \neq 0$. Since

$$
\pi_{\lambda}\left(X_{\alpha} X_{-\alpha}\right) v=\pi_{\lambda}\left(X_{-\alpha} X_{\alpha}\right) v+\pi_{\lambda}\left(H_{\alpha}\right) v=\lambda\left(H_{\alpha}\right) v=0
$$

and

$$
\pi_{\lambda}\left(H_{\alpha} X_{-\alpha}\right) v=\pi_{\lambda}\left(X_{-\alpha} H_{\alpha}\right) v+\pi_{\lambda}\left(-2 X_{-\alpha}\right) v=-2 \pi_{\lambda}\left(X_{-\alpha}\right) v,
$$

then $\pi_{\lambda}\left(X_{-\alpha}\right) v$ generates an infinite dimensional $s l_{2}$-submodule of $E^{\lambda}$, which contradicts the fact that $E^{\lambda}$ is finite dimensional.

Lemma 13. The highest weight vector $v \in E^{\lambda}$ is an eigenvector for the subgroup $K^{\lambda} \subset K$.

Since $K^{\lambda}$ is the integral subgroup of the subalgebra $k_{r}^{\lambda} \subset k_{r}$, it is enough to check the assertion of the lemma for the subalgebra $k_{r}^{\lambda}$. It is generated by the subalgebra $m_{r}$, for which $v$ is an eigenvector, and by the elements $X_{\alpha}-X_{-\alpha}$ and $i\left(X_{\alpha}+X_{-\alpha}\right), \alpha \in \triangle^{+}(\lambda)$, which annihilate $v$ according to Lemma 12 . 
Lemma 14. Let $f(k)$ be the covariant symbol of an operator $A \in E n d E^{\lambda}$. Then the function $f(k)$ is invariant under the right shifts by the elements of $K^{\lambda}$.

Proof. It follows from Lemma 13 and the unitarity of the representation of $K$ in $E^{\lambda}$ that $l v=c v$ for $l \in K^{\lambda}$, where $c$ is a constant of modulus 1 . Therefore, $f(k l)=\langle A k l v, k l v\rangle=\langle A k v, k v\rangle=f(k)$. The lemma is proved.

Theorem 2. All the functions from $A(\lambda+\rho), \lambda \in a_{r}^{*}$, are invariant under the right shifts by the elements of $K^{\lambda}$ iff $\lambda$ is relatively regular.

Proof. The necessity is proved in Lemma 11. Let us prove the sufficiency. Assume that $\lambda$ is regular relative to $\sigma \in \Sigma$. Denote by $a_{\sigma}^{*}$ the set of $\mu \in a_{r}^{*}$ such that $\mu\left(H_{\alpha}\right)=0$ for $\alpha \in \sigma$. In particular, $\lambda \in a_{\sigma}^{*}$. Denote by $\omega^{\alpha}$ the fundamental weight, corresponding to $\alpha \in \Sigma$ (i.e. $\omega^{\alpha}\left(H_{\beta}\right)=\delta_{\beta}^{\alpha}$ for $\alpha, \beta \in \Sigma$, where $\delta_{\beta}^{\alpha}$ is the Kronecker symbol). The fundamental weights $\omega^{\alpha}, \alpha \in \Sigma \backslash \sigma$, form a basis in $a_{\sigma}^{*}$, and their linear combinations with coefficients in $\mathbf{N}$ are the dominant weights, which are regular relative to $\sigma$. Consider $s_{\mu}(k)$ and $s_{\mu}(k l)$ for fixed $k \in K, l \in K^{\lambda}$ and $u \in \mathcal{U}\left(g_{c}\right)$ as polynomials in $\mu$ on $a_{\sigma}^{*}$. It follows from Proposition 2 and Lemma 14, that these polynomials coincide on the dominant weights $\mu$, which are regular relative to $\sigma$. It is easy to conclude from the above, that these polynomials coincide identically, which proves the theorem.

Corollary. Let $\Omega_{\lambda} \subset k_{r}$ be the adjoint orbit of the point $i H_{\lambda}$. The algebra $A(\lambda+\rho)$ can be pushed forward to $\Omega_{\lambda}$ by the mapping $\Psi_{\lambda}$ iff $\lambda$ is relatively regular.

\section{Quantization on FlaG MANifoldS}

Let $d$ be a nonnegative integer. Denote by $\mathcal{U}_{d}\left(g_{c}\right)$ the subspace of $\mathcal{U}\left(g_{c}\right)$ generated by all monomials of the form $X_{1} \ldots X_{j}$, where $X_{1}, \ldots, X_{j} \in g_{c}$ and $j \leq d$. The subspaces $\left\{\mathcal{U}_{d}\left(g_{c}\right)\right\}$ determine the canonical filtration on $\mathcal{U}\left(g_{c}\right)$ (see $[6]$ ).

The symmetric algebra $S\left(g_{c}\right)$ can be identified with the space of polynomials on $k_{r}$, so that an element $X \in g_{c}$ corresponds to the linear functional on $k_{r}$ given by $\tilde{X}(Y)=(X, Y), Y \in k_{r}$. Let $S^{d}\left(g_{c}\right)$ be the space of homogeneous polynomials on $k_{r}$ of degree $d$. The graded algebra associated with the canonical filtration is canonically isomorphic to $S\left(g_{c}\right)$, so that $\mathcal{U}_{d}\left(g_{c}\right) / \mathcal{U}_{d-1}\left(g_{c}\right)$ corresponds to $S^{d}\left(g_{c}\right)$. For $u \in \mathcal{U}_{d}\left(g_{c}\right)$ let $\underline{u}^{(d)}$ denote the corresponding element of $S^{d}\left(g_{c}\right)$. Usually we will omit the superscript $d$ in $\underline{u}^{(d)}$. If $u=X_{1} \ldots X_{d} \in \mathcal{U}_{d}\left(g_{c}\right)$, then $\underline{u}=\tilde{X}_{1} \ldots \tilde{X}_{d}$. We say that a monomial $u=X_{1} \ldots X_{d} \in \mathcal{U}\left(g_{c}\right)$ has canonical form, if for some integers $k, l$ such that $0 \leq k \leq l \leq d$, an element $X_{j}$ belongs to $n_{c}^{-}, h_{c}, n_{c}$ if $0<j \leq k, k<j \leq l, l<j \leq d$, respectively. Recall that the projection of $u \in \mathcal{U}\left(g_{c}\right)$ onto $\mathcal{U}\left(h_{c}\right)$ in (2) is denoted by $u_{0}$.

Proposition 3. Let $t \in \mathbf{R}, \lambda \in a_{r}^{*}, u \in \mathcal{U}_{d}\left(g_{c}\right), k \in K$. Then

$$
\lim _{t \rightarrow \infty} t^{-d} s_{t \lambda} u(k)=i^{-d} \underline{u}^{(d)}\left(\Psi_{\lambda}(k)\right) .
$$

Proof. Since $s_{\lambda}, \Psi_{\lambda}$ and the mapping $u \mapsto \underline{u}$ are $K$-equivariant, it is enough to check the equality in the proposition only at the point $e \in K$, i.e. one has to show that

$$
\lim _{t \rightarrow \infty} t^{-d} u_{0}(t \lambda)=i^{-d} \underline{u}\left(i H^{\lambda}\right) .
$$

Assume that $u \in \mathcal{U}_{d-1}\left(g_{c}\right)$. Then $u_{0}(t \lambda)$ is a polynomial in $t$ of degree less than $d$; hence the left-hand side in (7) is 0 . On the other hand $\underline{u}=0$, since $\mathcal{U}_{d-1}\left(g_{c}\right)$ is 
the kernel of the mapping $u \mapsto \underline{u}^{(d)}$. Now it remains to check (7) for a monomial $u=X_{1} \ldots X_{d} \in \mathcal{U}_{d}\left(g_{c}\right)$ which has canonical form. Since

$$
i^{-d} \underline{u}\left(i H^{\lambda}\right)=i^{-d} \tilde{X}_{1}\left(i H^{\lambda}\right) \ldots \tilde{X}_{d}\left(i H^{\lambda}\right)=\left(X_{1}, H^{\lambda}\right) \ldots\left(X_{d}, H^{\lambda}\right),
$$

the expression in (8) for $u \in \mathcal{U}\left(h_{c}\right)$ is equal to $u_{0}(\lambda)$. In this case $u_{0}(\lambda)$ is homogeneous of degree $d$, so the expression under the limit on the left-hand side of $(7)$ does not depend on $t$ and the left-hand side of (7) is also equal to $u_{0}(\lambda)$. Assume now that $u \notin \mathcal{U}\left(h_{c}\right)$. Then the factor $\left(X_{j}, H^{\lambda}\right)$ on the right-hand side of (8), for which $X_{j} \notin h_{c}$, is equal to 0 . On the other hand, in this case $u_{0}=0$, which concludes the proof.

Let $V$ be a $K$-module, $\nu \in a_{r}^{*}$ a dominant weight. Denote by $V^{\nu}$ the isotropic component of $V$, corresponding to the representation with the highest weight $\nu$. Let $\Omega \subset k_{r}$ be an adjoint orbit of the group $K$. Denote by $Q(\Omega)$ the space of $K$-finite functions on $\Omega$, and by $R(\Omega)$ the space of regular functions on $\Omega$, i.e., the space of restrictions of the functions from $S\left(g_{c}\right)$ on $\Omega$.

Lemma 15. (i) The spaces $Q(\Omega)$ and $R(\Omega)$ coincide.

(ii) The space $R(\Omega)^{\nu}$ for each dominant weight $\nu$ is finite dimensional.

(iii) There exist homogeneous polynomials $\psi_{1}, \ldots, \psi_{n} \in S\left(g_{c}\right)^{\nu}$ such that their restrictions on $\Omega$ form a basis in $R(\Omega)^{\nu}$.

Proof. Since each element in $S\left(g_{c}\right)$ is $K$-finite, $R(\Omega) \subset Q(\Omega)$. It follows from the Frobenius reciprocity theorem, that the irreducible representation of $K$ with the highest weight $\nu$ has a finite multiplicity in $Q(\Omega)$. Hence $Q(\Omega)^{\nu}$ and, therefore, $R(\Omega)^{\nu}$ are finite dimensional. Introduce a Hilbert space $L^{2}(\Omega, d x)$, where $d x$ is a $K$-invariant measure on $\Omega$. It contains $Q(\Omega)$. Assume $Q(\Omega)^{\nu} \backslash R(\Omega)^{\nu}$ is nonempty for some $\nu$. Then a function $f \in Q(\Omega)^{\nu}$ orthogonal to $R(\Omega)^{\nu}$ is orthogonal to the whole space $R(\Omega)$. It follows from the Stone-Weierstrass theorem, that $R(\Omega)$ is dense in $C(\Omega)$ and, therefore, in $L^{2}(\Omega, d x)$; thus $f=0$, which implies (i).

Finally, since $S\left(g_{c}\right)^{\nu}=\bigoplus\left(S^{d}\left(g_{c}\right)\right)^{\nu}$, the polynomials from (iii) can be constructed inductively.

Lemma 16. Let $\lambda \in a_{r}^{*}$ be a relatively regular element, $\nu \in a_{r}^{*}$ a dominant weight, $u \in \mathcal{U}_{d}\left(g_{c}\right)^{\nu}, t \in \mathbf{R},\left\{f_{1}, \ldots, f_{n}\right\}$ a basis in $R\left(\Omega_{\lambda}\right)^{\nu}$. Then $s_{t \lambda} u$ can be realized as a linear combination of functions $f_{j} \circ \Psi_{\lambda}, j=1, \ldots, n$, with coefficients which are polynomial in $t$ of a degree not greater than $d$.

Proof. It is easy to notice that for a given $\lambda$ the stabilizer $K^{t \lambda}$ coincides with $K^{\lambda}$ for all real $t \neq 0$, and with $K$ for $t=0$. Since $t \lambda$ is relatively regular for all $t$, it follows from Theorem 2 that $s_{t \lambda} u$ is the pullback of some function $\psi_{t}$ on $\Omega_{\lambda}$, that is, $s_{t \lambda} u=\psi_{t} \circ \Psi_{\lambda}$. The $K$-equivariance of $s_{\lambda}$ and Lemma 15 imply that $\psi_{t} \in R\left(\Omega_{\lambda}\right)^{\nu}$; thus there exist functions $a_{j}(t), j=1, \ldots, n$, such that $\psi_{t}=\sum a_{j}(t) f_{j}$. Since $R\left(\Omega_{\lambda}\right)^{\nu}$ is $n$-dimensional, there exist points $x_{1}, \ldots, x_{n} \in \Omega_{\lambda}$ such that the matrix $\left(f_{i}\left(x_{j}\right)\right)$ is invertible. Let $\left(b_{i j}\right)$ be its inverse; then $a_{i}(t)=\sum b_{i j} \psi_{t}\left(x_{j}\right)$. Now the assertion of the lemma follows from the fact that for each $k \in K$ the function $\psi_{t}\left(\Psi_{\lambda}(k)\right)=s_{t \lambda} u(k)$ is polynomial in $t$ of degree not greater than $d$.

Let $\beta: S\left(g_{c}\right) \rightarrow \mathcal{U}\left(g_{c}\right)$ be the symmetrization mapping (see [6]). It is $K$ equivariant, and for a homogeneous polynomial $\psi \in S^{d}\left(g_{c}\right)$ we have $\beta \psi \in \mathcal{U}_{d}\left(g_{c}\right)$ and $\beta \psi=\psi$. 
Lemma 17. Let $\lambda \in a_{r}^{*}$ be relatively regular, $f \in R\left(\Omega_{\lambda}\right)$. There exist $u_{j} \in$ $\mathcal{U}_{d(j)}\left(g_{c}\right), j=1, \ldots, m$, and rational functions $\left\{a_{j}(t)\right\}$ with no poles at infinity, such that for all $t \neq 0$ different from the poles of the functions $\left\{a_{j}(t)\right\}$, we have

$$
f \circ \Psi_{\lambda}=\sum a_{j}(t) t^{-d(j)} s_{t \lambda} u_{j} .
$$

Proof. It is enough to prove the lemma for functions $f_{1}, \ldots, f_{n}$ which form a basis of $R\left(\Omega_{\lambda}\right)^{\nu}$ for an arbitrary $\nu$. According to Lemma 15, one can assume that $f_{j}$ is a restriction of a homogeneous polynomial $\psi_{j}$ of the degree $d(j)$. It follows from Lemma 16 that there exist polynomials $b_{j l}(t)$ of degree not greater than $d(j)$, such that

$$
\left(\frac{i}{t}\right)^{d(j)} s_{t \lambda} \beta \psi_{j}=\sum_{l} t^{-d(j)} b_{j l}(t) f_{l} \circ \Psi_{\lambda} .
$$

Using Proposition 3 and the properties of $\beta$, pass to the limit on both sides of (9) as $t \rightarrow \infty$. Since the left-hand side of (9) tends to $f_{j} \circ \Psi_{\lambda}$, then the matrix of rational functions $\left(t^{-d(j)} b_{j l}(t)\right)$ tends to the identity matrix as $t \rightarrow \infty$. Therefore its determinant is not identically zero, and its inverse matrix of rational functions, $\left(a_{l j}(t)\right)$ exists, which also tends to the identity matrix as $t \rightarrow \infty$. The assertion of the lemma is obtained by applying the matrix $\left(a_{l j}(t)\right)$ to both sides of $(9)$ and setting $u_{j}=i^{d(j)} \beta \psi_{j}$.

It is known that there exists a natural Poisson structure on the symmetric algebra $S\left(g_{c}\right)$, which is considered as the algebra of polynomials on $k_{r}$. It is defined by the (real) Poisson bracket $\{\cdot, \cdot\}$ on $k_{r}$ such that for $X, Y \in g_{c}$ and $Z=[X, Y]$, we have $\{\tilde{X}, \tilde{Y}\}=\tilde{Z}$. The symplectic leaves of that Poisson structure are the (co)adjoint orbits of $K$ in $k_{r}$ (the Lie algebra $k_{r}$ is identified with its dual space $k_{r}^{*}$ via the Killing form). The symplectic structure on a (co)adjoint orbit $\Omega \subset k_{r}$ is defined by the Kirillov symplectic form, and the corresponding Poisson bracket $\{\cdot, \cdot\}_{\Omega}$ is the restriction of $\{\cdot, \cdot\}$ on $\Omega$, i.e. for $f, g \in S\left(g_{c}\right)$ we have $\left.\{f, g\}\right|_{\Omega}=\left\{\left.f\right|_{\Omega},\left.g\right|_{\Omega}\right\}_{\Omega}$ (see $[7])$.

The multiplication and the Poisson bracket on $S\left(g_{c}\right)$ are connected with the canonical filtration on $\mathcal{U}\left(g_{c}\right)$ in the following way (see [6]). Let $u_{\varepsilon} \in \mathcal{U}_{d_{\varepsilon}}\left(g_{c}\right), \varepsilon=$ $1,2, d=d_{1}+d_{2}$. Then $u_{1} u_{2} \in \mathcal{U}_{d}\left(g_{c}\right), u_{1} u_{2}-u_{2} u_{1} \in \mathcal{U}_{d-1}\left(g_{c}\right)$, and

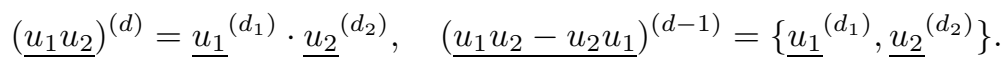

Let $\lambda \in a_{r}^{*}$ be relatively regular, $t \in \mathbf{R} \backslash\{0\}$. It follows from the fact that $K^{t \lambda}=K^{\lambda}$, and Theorem 2, that the algebra $A(t \lambda+\rho)$ can be pushed forward to the orbit $\Omega_{\lambda}$ via $\Psi_{\lambda}$. Denote by $\mathcal{A}_{1 / t}^{(\lambda)}$ and $*_{1 / t}^{(\lambda)}$ the pushforward of $A(t \lambda+\rho)$ on $\Omega_{\lambda}$, and the multiplication in it, respectively. Usually the superscript $\lambda$ in these notations will be omitted. Since each element in $A(t \lambda+\rho)$ is $K$-finite, Lemma 15 implies that $\mathcal{A}_{1 / t}^{(\lambda)} \subset R\left(\Omega_{\lambda}\right)$.

Theorem 3. Let $\lambda \in a_{r}^{*}$ be relatively regular, $t \in \mathbf{R} \backslash\{0\}$.

(i) Each function $f \in R\left(\Omega_{\lambda}\right)$ belongs to $\mathcal{A}_{1 / t}^{(\lambda)}$ for all but a finite number of values of $t$.

(ii) For $f_{1}, f_{2} \in R\left(\Omega_{\lambda}\right), x \in \Omega_{\lambda}$, the product $\left(f_{1} *_{1 / t} f_{2}\right)(x)$ is a rational function of $t$, with no pole at infinity.

(iii) For $f_{1}, f_{2} \in R\left(\Omega_{\lambda}\right)$ the following limits hold:

$$
\lim _{t \rightarrow \infty} f_{1} *_{1 / t} f_{2}=f_{1} f_{2} ; \quad \lim _{t \rightarrow \infty} t\left(f_{1} *_{1 / t} f_{2}-f_{2} *_{1 / t} f_{1}\right)=i\left\{f_{1}, f_{2}\right\}_{\Omega_{\lambda}} .
$$


Proof. Assertion (i) follows directly from Lemma 17. In accordance with Lemma 17, there exist the elements $u_{\varepsilon j} \in \mathcal{U}_{d_{\varepsilon}(j)}\left(g_{c}\right), \varepsilon=1,2, j=1, \ldots, n(\varepsilon)$, and rational functions $a_{\varepsilon j}(t)$, with no poles at infinity, such that for all $t \neq 0$, different from the poles of $\left\{a_{\varepsilon j}(t)\right\}$,

$$
f_{\varepsilon} \circ \Psi_{\lambda}=\sum_{j=1}^{n(\varepsilon)} a_{\varepsilon j}(t) t^{-d_{\varepsilon}(j)} s_{t \lambda} u_{\varepsilon j} .
$$

Passing to the limit in (11) as $t \rightarrow \infty$, and denoting $\lim _{t \rightarrow \infty} a_{\varepsilon j}(t)=a_{\varepsilon j}$, one gets from Proposition 3, that

$$
f_{\varepsilon} \circ \Psi_{\lambda}=\sum_{j} a_{\varepsilon j} i^{-d_{\varepsilon}(j)} \underline{u}_{\varepsilon j} \circ \Psi_{\lambda} .
$$

Let us calculate the product $f_{1} *_{1 / t} f_{2}$, pulling it back to $K$ via $\Psi_{\lambda}$ :

$$
\left(f_{1} *_{1 / t} f_{2}\right) \circ \Psi_{\lambda}=\sum_{j, l} a_{1 j}(t) a_{2 l}(t) t^{-d(j, l)} s_{t \lambda}\left(u_{1 j} u_{2 l}\right),
$$

where $d(j, l)=d_{1}(j)+d_{2}(l)$. Since $u_{1 j} u_{2 l} \in \mathcal{U}_{d(j, l)}\left(g_{c}\right)$, for each $k \in K$ the function $s_{t \lambda}\left(u_{1 j} u_{2 l}\right)(k)$ is a polynomial in $t$ of a degree not greater than $d(j, l)$. Thus each summand in (13) is a rational function of $t$, with no pole at infinity, which proves (ii).

Pass to the limit in (13) as $t \rightarrow \infty$, using Proposition 3 and taking into account (10), (11) and (12):

$$
\begin{aligned}
& \lim _{t \rightarrow \infty}\left(f_{1} *_{1 / t} f_{2}\right) \circ \Psi_{\lambda}=\sum_{j, l} a_{1 j} a_{2 l} i^{-d(j, l)}\left(\underline{u_{1 j} u_{2 l}}\right) \circ \Psi_{\lambda} \\
& =\left(\sum_{j, l} a_{1 j} a_{2 l} i^{-d_{1}(j)} \underline{u}_{1 j} i^{-d_{2}(l)} \underline{u}_{2 l}\right) \circ \Psi_{\lambda}=\left(f_{1} f_{2}\right) \circ \Psi_{\lambda} .
\end{aligned}
$$

Thus we obtain the first of the two limits in (iii). The second limit is calculated similarly:

$$
\begin{aligned}
\lim _{t \rightarrow \infty} & t\left(f_{1} *_{1 / t} f_{2}-f_{2} *_{1 / t} f_{1}\right) \circ \Psi_{\lambda} \\
& =\lim _{t \rightarrow \infty} \sum_{j, l} a_{1 j}(t) a_{2 l}(t) t^{-d(j, l)+1} s_{t \lambda}\left(u_{1 j} u_{2 l}-u_{2 l} u_{1 j}\right) \\
& =\sum_{j, l} a_{1 j} a_{2 l} i^{-d(j, l)+1}\left(\underline{u_{1 j} u_{2 l}-u_{2 l} u_{1 j}}\right) \circ \Psi_{\lambda} \\
& =i\left(\sum_{j, l} a_{1 j} a_{2 l}\left\{i^{-d_{1}(j)} \underline{u}_{1 j}, i^{-d_{2}(l)} \underline{u}_{2 l}\right\}\right) \circ \Psi_{\lambda}=i\left\{f_{1}, f_{2}\right\}_{\Omega_{\lambda}} \circ \Psi_{\lambda} .
\end{aligned}
$$

Remark 1 . The assertion (i) of Theorem 3 means, that the algebras $\mathcal{A}_{1 / t}^{(\lambda)}$ have the weak nesting property. It is proved in [8] that if $\lambda$ is a dominant weight and $n \in \mathbf{N}$, then the $\mathcal{A}_{1 / n}^{(\lambda)} \subset \mathcal{A}_{1 /(n+1)}^{(\lambda)}$, i.e., the algebras of covariant symbols, $\left\{\mathcal{A}_{1 / n}^{(\lambda)}\right\}$, have the strong nesting property.

Remark 2. If $\lambda$ is a dominant weight, the assertion (ii) of Theorem 3 is proved when $\Omega_{\lambda}$ is a compact hermitian symmetric space, and conjectured for an arbitrary generalized flag manifold in [3]. 
Remark 3. The assertion (iii) of Theorem 3 is proved in [3] for the case when $\lambda$ is a dominant weight.

Remark 4. The connection between the $K$-orbit of the highest weight vector $v \in E^{\lambda}$ and the adjoint orbit $\Omega_{\lambda}$ with their application to covariant symbols is studied in [4].

Now we can define Berezin's quantization on a generalized flag manifold, using the algebras of mixed symbols. Assume $\lambda \in a^{*}$ is a relatively regular weight, and there exists an element $w \in W$ such that the weights $w \lambda$ and $w \cdot \lambda$ are dominant. Then for all natural $n$ the weights $w \cdot(n \lambda)=(n-1) w \lambda+w \cdot \lambda$ are dominant. The algebra $A(n \lambda+\rho)$ has the representation $\tau_{n \lambda}$ in the $g_{c}$-module $E^{w \cdot(n \lambda)}$. Pushing forward $A(n \lambda+\rho)$ together with its representation $\tau_{n \lambda}$ to $\Omega_{\lambda}$, we obtain the algebra $\mathcal{A}_{1 / n}^{(\lambda)}$ with a representation in $E^{w \cdot(n \lambda)}$. Denote $\Omega=\Omega_{\lambda}, F=\{1,1 / 2,1 / 3, \ldots\}, h=$ $1 / n \in F, \mathcal{A}_{h}=\mathcal{A}_{1 / n}^{(\lambda)}, H_{h}=E^{w \cdot(n \lambda)}$. Then Theorem 3 implies

Theorem 4. The algebras $\left\{\mathcal{A}_{h}\right\}, h \in F$, together with their representations in $H_{h}$ define Berezin's quantization on the (co)adjoint orbit $\Omega$ of the group $K$, which is a generalized flag manifold.

\section{ACKNOWLEDGEMENTS}

I wish to express my gratitude to Professors M.S. Narasimhan and D. Vogan for helpful discussions. I also thank the International Centre for Theoretical Physics in Trieste, Italy, the European School in Group Theory, and the University of Odense, Denmark, for their hospitality.

\section{REFERENCES}

[1] F.A.Berezin, Quantization on bounded complex domains, Soviet Math. Dokl., 14(1973), 12091213. MR 48:10346

[2] F.A.Berezin, General concept of quantization, Comm. Math. Phys. 40 (1975), 153-174. MR 53:15186

[3] M.Cahen, S.Gutt, J.Rawnsley, Quantization of Kähler manifolds II, TAMS, 337 (1993), 73-98. MR 93i: 58063

[4] N.Wildberger, On the Fourier transform of a compact semisimple Lie group, J. Austral. Math. Soc. Ser. A 56 (1994), 64-116. MR 95g:22026

[5] M.Duflo, Representations irreductibles des groupes semi-simples complexes, Lecture Notes in Math., vol. 497, Springer-Verlag, Berlin, Heidelberg and New York, 1975, pp. 26-88. MR 53:3198

[6] J.Dixmier, Algèbres enveloppantes, Gauthier-Villars, Paris, 1974. MR 58:16803a

[7] F.Bayen et al., Deformation theory and quantization, Ann. Phys. 111 (1978) 61-151. MR 58:14737a,b

[8] J.Rawnsley, M.Cahen, S.Gutt, Quantization of Kähler manifolds I: Geometric interpretation of Berezin's quantization, J. Geom. Phys. 7 (1990), 45-62. MR 92e:58082

LCta, Joint Institute for Nuclear Research, Dubna 141980, Moscow Region, Russia

E-mail address: karabegov@vxjinr.jinr.ru 